\title{
Structural and Functional Brain Changes in
}

\section{Hemodialysis Patients with End-Stage Renal Disease: DTI Analysis Results and ALFF Analysis Results}

\author{
Huiying Guo $\mathbb{D}^{1, *}$ \\ Wenjin $\mathrm{Liu}^{2, *}$ \\ Haige $\mathrm{Li}^{\prime}$ \\ Junwei Yang ${ }^{2}$
}

'Department of Radiology, Second Affiliated Hospital of Nanjing Medical University, Nanjing, Jiangsu, People's Republic of China; ${ }^{2}$ Center for Kidney Disease, Second Affiliated Hospital of Nanjing Medical University, Nanjing,

Jiangsu, People's Republic of China

*These authors contributed equally to this work
This article was published in the following Dove Press journal:

International Journal of Nephrology and Renovascular Disease

Introduction: The current study aimed to depict intrinsic structural changes and the spontaneous brain activity patterns in voxel level in patients with end-stage renal disease (ESRD) undergoing hemodialysis (HD) by using diffusion-tensor imaging and resting-state functional magnetic resonance (MR) imaging with an amplitude of low-frequency fluctuations (ALFF) algorithm and their clinical relevance.

Materials and Methods: In the study, the diffusion-tensor imaging and resting-state functional MR imaging were performed in forty-two hemodialysis patients with ESRD and 42 healthy control subjects. Neuropsychological and laboratory tests were performed in all subjects. ALFF, fraction anisotropy (FA), and mean diffusivity (MD) values were compared between the two groups. Correlations between ALFF, FA or MD values, and clinical markers were analyzed.

Results: We found that ESRD patients exhibited significantly lower ALFF values in multiple areas, including medial frontal gyrus, limbic lobe, superior frontal gyrus, bilateral lingual gyri, occipital lobe, parahippocampal gyrus, precuneus, while increased ALFF values in medial frontal gyrus than healthy controls. FA values were decreased in medial frontal gyrus, parietal lobe, and left precuneus regions in the ESRD group compared with controls. Importantly, FA for the frontal and parietal lobes was negatively associated with the dialysis duration of ESRD patients, ALFF z-scores for the medial prefrontal cortex (MPFC) were positively correlated with the dialysis duration of ESRD patients and Serum calcium of ESRD patients negatively correlated with FA values in the frontal and parietal lobes $(p<0.05)$.

Conclusion: Our study revealed that both impaired brain structure and function in ESRD patients with routine hemodialysis distributed mainly in the parietal, temporal, and frontal lobes. ESRD patients have cognitive impairment and declined memory ability. Serum calcium and dialysis duration might be associated with the impairment of brain structure and function in patients with ESRD.

Keywords: cognitive impairment, hemodialysis, end-stage renal disease, resting-state functional MRI, diffusion-tensor imaging

\section{Introduction}

End-stage renal disease (ESRD) has been shown to not only be accompanied by central nervous system abnormalities (eg white matter lesions, cerebral atrophy, and myelinoclasis) ${ }^{1}$ but also results in various neurological problems (eg dialysis
Department of Radiology, Second

Affiliated Hospital of Nanjing Medical

University, Nanjing, 210003, People's

Republic of China

Email lihaige@njmu.edu.cn

Junwei Yang

Center for Kidney Disease, Second Affiliated Hospital of Nanjing Medical University, Nanjing, 210003, People's Republic of China

Email jwyang@njmu.edu.cn

International Journal of Nephrology and Renovascular Disease 2021:|4 77-86 
encephalopathy, uremic encephalopathy). ${ }^{2}$ Moreover, ESRD patients have a higher risk for developing cognitive impairments and ultimately death. ${ }^{3,4}$ Therefore, early assessment of brain abnormalities in neurologically asymptomatic patients with ESRD is crucial for prompt treatment and the improvement of patients' prognosis. Approximately $75 \%$ of the patients who are receiving dialysis exhibit cognitive impairments. The impairments involve a range of cognitive domains, including concentration, memory, and planning, which may be associated with more frequent and longer hospitalizations. ${ }^{5,6}$ The underlying pathophysiology of this cognitive dysfunction in the ESRD patients undergoing hemodialysis (HD) remains unclear.

Recently, neuroimaging techniques have been established as a valuable tool to investigate the neuropathologic mechanisms underlying ESRD-related neurological complications. $^{2,7-9}$ Insights from resting-state functional Magnetic Resonance imaging (rs-fMRI) have suggested that the pathophysiology of various mental disorders in humans is associated with changes of spontaneous lowfrequency blood-oxygen-level-dependent (BOLD) signal fluctuations $(<0.08 \mathrm{~Hz})$ in the brain. ${ }^{10}$ rs-fMRI is a reliable tool for investigating neural networks. The amplitude of low-frequency fluctuation (ALFF) is a method that has been used to evaluate local spontaneous neuronal activity in rs-fMRI analysis based on BOLD signals. It reflects the amplitude of neuronal activity within a single voxel by calculating the square root of the power spectrum in a frequency range (typically $0.01-0.08 \mathrm{~Hz}$ ). ${ }^{10}$ Previous studies have shown the specific patterns of ALFF in patients with ESRD who were undergoing peritoneal dialysis (PD) that correlate with cognitive impairments. ${ }^{11}$ However, to our knowledge, only a few rs-fMRI studies $^{12-14}$ have been performed in ESRD patients. These studies did not investigate the effect of the specific dialysis modality on spontaneous brain activity in patients with ESRD, as they included patients undergoing HD and peritoneal dialysis and also those not undergoing dialysis. This can have a confounding effect on the study results.

In addition, most previous studies have been focused on abnormal gray and white brain matter structures. ${ }^{9,14-18}$ The studies showed that compared with normal controls, not only the local microstructure of white matter integrity ${ }^{16,17}$ but also the interregional fiber tractography ${ }^{18}$ is changed in patients with ESRD. Zhang et $\mathrm{al}^{19}$ using diffusion-tensor imaging (DTI) and rs-fMRI to investigate structural and functional alterations of the default mode network (DMN) in the brain after renal transplantation in patients with end-stage renal disease, found that functional connectivity changes in the DMN, which were associated with improved hematocrit levels and cognitive function, may recover earlier than structural connectivity changes do 1 month after renal transplantation. DTI, a non-invasive tool for probing the microanatomical organization of human brain white matter in vivo, has been used to investigate the property of white matter in ESRD. Fractional anisotropy (FA), an index derived from DTI, which can be used to evaluate the extent of anisotropy in tissues, mainly reflecting the integrity of fiber bundle. Higher FA value represents greater diffusion anisotropy. For Mean diffusivity (MD) the dispersion level and resistance of the whole molecule can be reflected, regardless of the direction of dispersion. Larger MD indicates more free water molecules in the tissue. ${ }^{20-22}$

To our knowledge, no study has combined DTI with rsfMRI to investigate the fiber bundle impairment and functional cerebral deficits simultaneously in ERSD. This method could have the potential to explore the neurobiological mechanisms of ESRD patients.

We hypothesized that HD had a negative effect on resting-state spontaneous brain activity and abnormalities on the diffusion tensor function in ESRD patients. To verify this hypothesis, we applied both DTI and rs-fMRI in 42 ESRD patients and 42 control subjects to analyze the changes in structural connectivity and spontaneous brain activity patterns in ESRD patients who were undergoing HD.

\section{Materials and Methods \\ Subjects}

A total of forty-two patients with ESRD (22 males and 20 females with mean age $51 \pm 10.2$ years) on maintenance HD (three times per week) for at least 3 months, all righthanded, were enrolled. All patients were recruited from the dialysis center of Second Affiliated Hospital of Nanjing Medical University. Exclusion criteria included: history of drug or alcohol abuse, obvious brain lesions such as stroke and tumor, a history of Parkinson's or neurodegenerative disease, psychiatric disorder, or major neurologic disorders (severe head injury, visible lesions, epilepsy, or stroke), and any contraindications to MR imaging.

Forty-two controls (22 males and 20 females with mean age $50 \pm 10.0$ years; all right-handed) were recruited from the local community. None of the controls had any 
kidney disease or history of neurological or psychiatric diseases. We used the same exclusion criteria as those used for the ESRD patients.

\section{Laboratory Examinations and Neuropsychological Tests}

Previous work reported that studies performed during or immediately after hemodialysis could induce adverse acute effects on brain physiology and cognitive function, ${ }^{23-27}$ which we sought to avoid. Thus, for our study, cognitive function and MRI experiments were performed at least 24 $\mathrm{h}$ after a hemodialysis session. All participants were required to refrain from food or drink containing caffeine for at least $8 \mathrm{~h}$ before the MRI exam or cognitive assessment. ${ }^{28}$ Each MRI or cognitive assessment study for each participant was scheduled at a similar time of day. All participants were instructed to complete the Beijing version of Montreal Cognitive Assessment (MoCA), under the supervision of a physician. The subsequent neuropsychological battery was tested in memory cognition domains by the Auditory Verbal Learning Test (AVLT) delay recall. ${ }^{29}$ The clinical data including duration of hemodialysis, the evaluation of systolic and diastolic blood pressure were also collected. The ESRD group completed multiple biochemical tests after the hemodialysis (within 36 hours) but before the MR imaging (within 24 hours). The biochemical tests included lipoprotein, cholesterol, hemoglobin, serum phosphorus, and serum calcium.

\section{MR Imaging}

Imaging data were acquired on a 3 Tesla MR scanner (GE Healthcare, signaHDxt). All of the patients were instructed to hold still, keep their eyes closed but be awake in the MR scanner. During the scan, the participants were fitted to foam padding to reduce head motion. The T2-FLAIR sequence and T1-weighted sequence images were obtained for every subject to exclude brain anatomical lesions. A gradient-echo echo-planar sequence was used to acquire BOLD rs-fMRI data. The parameters were as follows: repetition time $(\mathrm{TR})=2000 \mathrm{~ms}$ (echo time) $\mathrm{TE}=30 \mathrm{~ms}$, slice thickness $=4.0 \mathrm{~mm}$, slice gap $=0.4 \mathrm{~mm}$, FOV $=$ $240 \times 240 \mathrm{~mm}^{2}$, matrix $=64 \times 64$, flip angle $=90^{\circ}$, number of slices $=32$, and 250 volumes. Each fMRI scan lasted 500s. Besides, a spin-echo echo-planar imaging sequence was performed to acquire diffusion-tensor images. A diffusion gradient was applied in 20 noncollinear directions with $\mathrm{b}$ values of 0 and $1000 \mathrm{sec} / \mathrm{mm}^{2}(\mathrm{TR}=$ $13500 \mathrm{~ms}, \mathrm{TE}=87 \mathrm{~ms}, \mathrm{FOV}=240 \times 240 \mathrm{~mm}^{2}$, matrix $=$ $130 \times 128$, slice thickness $=3 \mathrm{~mm}$; no intersection gap).

\section{Data Preprocessing}

BOLD-fMRI data were pre-processed with the Data Processing and Analysis of Brain Imaging software ${ }^{30}$ running in MATLAB (MathWorks, Natick, MA, USA). The first 10 volumes were discarded to allow the MR signal to reach a steady-state and to allow the participants to adapt to the scanner environment. The pre-processing steps included a slice-timing correction, realignment of the functional data to each participant's first images, coregistration of the functional to structural images, and spatial normalization to that of the Montreal Neurological Institute (MNI, resampled voxel size $=3 \times$ $3 \times 3 \mathrm{~mm}^{3)}$ were conducted. After normalization, a bandpass filter was applied to maintain low-frequency fluctuations within a frequency range of $0.01-0.08 \mathrm{~Hz}$. Finally, subjects with head movement $>2 \mathrm{~mm}$ were excluded from the analysis. Several nuisance covariates (friston 24 head motion parameters, the average signals of cerebrospinal fluid and white matter) were regressed out from the data.

\section{ALFF Analysis}

The ALFF analysis was performed based on a previous study. ${ }^{31}$ The time sequences for a given voxel were transformed to the frequency series by Fast Fourier Transform (FFT), and the square root of the power spectrum was calculated and filtered across $0.01-0.08 \mathrm{~Hz}$. The average square root was considered to be the ALFF value. To reduce individual differences among subjects, the average ALFF value was subtracted from the ALFF value of each voxel and divided by the global mean ALFF value for standardization purposes.

\section{DTI Analysis}

The DTI imaging data were preprocessed with Diffusion Toolkit, a set of command-line tools with a GUI frontend that performs data reconstruction and fiber tracking on diffusion MR images (http://www.trackvis.org/dtk). The workflow of Diffusion Toolkit (DTK) contained 2 major steps: (1) convert the raw DICOM images into a series of ANALYZE images and (2) reconstruct the raw images. Then, the MD and FA functional maps were processed and analyzed by the software of SPM8 (based on MATLAB). The B0 maps of each subject were co-registered with the EPI template in SPM8 to the MNI standard space. Then, the MD 
and FA maps were standardized with the co-registered B0 maps. Finally, the registered FA and MD maps were smoothed with an isotropic Gaussian kernel (8-mm fullwidth at half maximum).

\section{Statistics Analysis}

All of the demographic and clinical data were analyzed by utilizing SPSS 17.0 (SPSS Inc., Chicago, IL). The Kolmogorov-Smirnov test indicated that not all the data showed normality. Therefore, the years of education, cognitive test results (AVLT, MoCA) were compared by nonparametric tests. A two-sample $t$-test was used to compare the age, systolic blood pressure, diastolic blood pressure and hemoglobin. Chi-square tests were used for the gender of the two groups. The ALFF, FA and MD values between patient and control groups were compared using voxelwise two-sample t-tests with age and gender as covariates by using Statistical parametric mapping software (SPM8; The FIL Methods Group, London, England). To check for multiple comparisons in the statistical analysis, we used the AlphaSim correction to adjust the alpha level $(p<0.05$ and cluster size of at least 66 voxels, AlphaSim corrected, Analysis of Functional NeuroImages, http://afni.nimh.nih. gov/afni/). ${ }^{32}$ The surviving clusters were reported.

A Spearman correlation analysis was conducted to observe the possible clinical relevance between changes in brain function and all clinical biochemical markers of
ESRD. Individual mean ALFF, FA and MD z-scores for the surviving clusters of ESRD patients were extracted for a Spearman correlation analysis. These correlations were adjusted for age and gender as covariates. Significant correlations were determined based on $p$-values less than 0.05 .

\section{Results}

An initial group of 60 ESRD patients was recruited from the dialysis center of Second Affiliated Hospital of Nanjing Medical University. All hemodialysis patients successfully completed both cognition assessment and MRI sessions. In addition, the data of eighteen patients were excluded resulting from excessive motion during MRI exams. Therefore, a total of 42 hemodialysis patients were included for final data analysis. A total of 42 age- and sex-matched controls were recruited accordingly.

\section{Demographic Results}

Demographic, clinical data and the biochemical results of the participants are shown in Table 1. The distributions of gender, age, and education were matched between the two groups. Systolic and diastolic blood pressure values did not differ between the ESRD and control groups. Significant differences were found in hemoglobin level, MoCA and AVLT scores $(p<0.001)$.

Table I The Demographic, Biochemical and Clinical Characteristics for All the Participants

\begin{tabular}{|c|c|c|c|}
\hline Demographic & ESRD & Controls & $p$ value \\
\hline Gender* (Male/Female) & $22 / 20$ & $22 / 20$ & $0.663^{\mathrm{a}}$ \\
\hline Age (years) & $50.93 \pm 10.17$ & $50.43 \pm 9.97$ & $0.821^{b}$ \\
\hline Education** (years) & $12(3)$ & $9(3)$ & $0.625^{c}$ \\
\hline MoCA** (score) & $22.5(5.25)$ & $25.5(4.25)$ & $<0.001^{c}$ \\
\hline AVLT-delay recall** & $5.07(2.00)$ & $7.07(3.25)$ & $<0.001^{c}$ \\
\hline Systolic blood pressure & $130.56 \pm 25.46$ & $125.87 \pm 15.53$ & $0.312^{b}$ \\
\hline Diastolic blood pressure & $85.82 \pm 12.88$ & $83.14 \pm 10.27$ & $0.295^{b}$ \\
\hline Hemoglobin $(g / L)$ & $108.12 \pm 14.58$ & $145.83 \pm \mid 8.37$ & $<0.001^{b}$ \\
\hline Dialysis duration (months) & $113.02 \pm 78.08$ & & \\
\hline Serum calcium (mmol/L) & $2.46 \pm 0.24$ & & \\
\hline Serum Phosphorus (mmol/L) & $1.54 \pm 0.39$ & & \\
\hline
\end{tabular}

Notes: Unless otherwise indicated, data are represented as mean \pm standard deviation. *Data are number of patients or control subjects. **Data are median (Quartile distance). ${ }^{\mathrm{a}}$ The $p$ value was obtained by chi-square test. ${ }^{\mathrm{b}}$ The $p$ values were obtained by two-side two-sample t-tests. ${ }^{\mathrm{c}}$ The $p$ values were obtained by nonparametric tests. Abbreviations: MoCA, Montreal Cognitive Assessment; AVLT: Auditory Verbal Learning Test. 


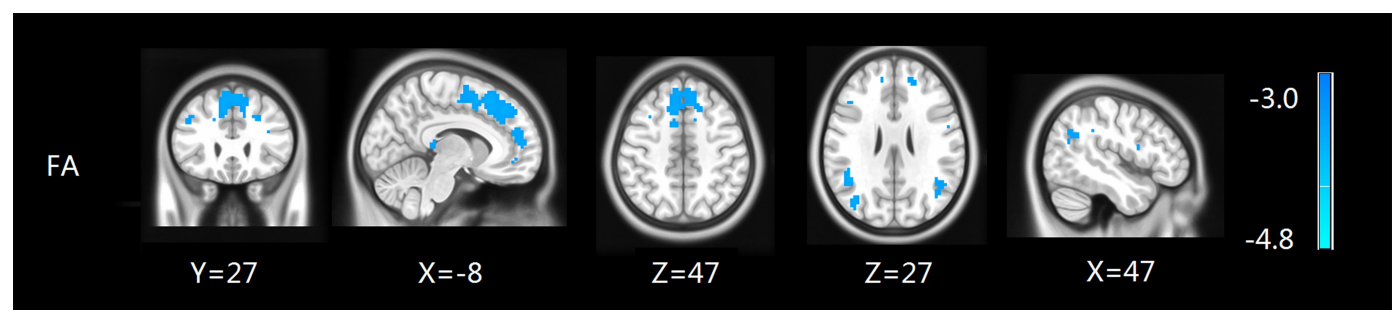

Figure I FA maps show differences between ESRD and the control groups ( $p<0.05$, AlphaSim corrected). The patients with ESRD showed a significant FA decrease in the medial frontal gyrus, parietal lobe, supramarginal gyrus, inferior parietal gyrus and middle temporal gyrus.

\section{DTI Analysis}

Figure 1 illustrates the results of the two-sample analysis of FA and MD between the two groups. Compared with the control group, the patients with ESRD showed a significant FA decrease in the medial frontal gyrus, parietal lobe, supramarginal gyrus, inferior parietal gyrus and middle temporal gyrus ( $p<0.05$, AlphaSim corrected), while no significant findings were found in MD values between the two groups (Table 2 and Figure 1).

\section{ALFF Analysis}

Compared to the control group, ESRD patients showed a significant ALFF decrease in the medial frontal gyrus, parietal lobe, inferior parietal gyrus, supramarginal gyrus, right lingual gyrus, and parahippocampal gyrus, but an increase in the bilateral superior frontal gyrus as shown in Table 3 and Figure 2 ( $p<0.05$, AlphaSim corrected). We also calculated the frame-wise displacement (FD) at each time point using 6 displacements from the rigid body motion correction procedure. ${ }^{33,34}$ There were no significant differences in the FD values between the groups $(p>0.05)$.

\section{Correlation Analysis}

As shown in Figure 3, the duration of hemodialysis of patients negatively correlated with mean $z$ scores of FA in the medial frontal gyrus, parietal lobe $(r=-0.326, p<0.05)$ (Figure 3A). The duration of hemodialysis had a positive relationship with mean $z$ scores of ALFF in medial frontal gyrus $(r=0.312, p<0.05)$ (Figure 3B). Serum calcium of ESRD patients negatively correlated with FA values in the frontal and parietal lobes $(r=-0.319, p<0.05)$ (Figure 3C). There was no significant correlation between FA, ALFF values and other clinical and biochemical markers.

\section{Discussion}

By combining both ALFF and DTI approaches to detect functional and structural plasticity in patients with ESRD, this study revealed the following important findings.

Table 2 Regions Showing FA Differences Between ESRD and Control Groups

\begin{tabular}{|c|c|c|c|c|c|c|}
\hline \multirow[t]{2}{*}{ Brain Regions } & \multirow[t]{2}{*}{ Brodman Area } & \multicolumn{3}{|c|}{ MNI Coordinates $(\mathrm{mm})$} & \multirow[t]{2}{*}{ Volume $\left(\mathrm{mm}^{3}\right)$} & \multirow[t]{2}{*}{ Peak $t$ Value } \\
\hline & & $\mathbf{x}$ & y & $\mathbf{z}$ & & \\
\hline Medial frontal gyrus & 8 & -10 & 17 & 51 & 800 & 3.48 \\
\hline Right parietal lobule & 39 & 48 & -61 & 22 & 69 & 2.66 \\
\hline Right supramarginal gyrus & 40 & 48 & -57 & 30 & 33 & 3.04 \\
\hline Left parietal lobule & 40 & -48 & -51 & 30 & 217 & 3.54 \\
\hline Left supramarginal gyrus & 39 & -44 & -52 & 24 & 90 & 3.12 \\
\hline Left inferior parietal gyrus & 40 & -39 & -35 & 34 & 88 & 2.98 \\
\hline Left superior frontal gyrus & 8 & 10 & 29 & 51 & 727 & 3.48 \\
\hline Left middle temporal gyrus & 39 & -39 & -75 & 24 & 351 & 3.12 \\
\hline Left middle occipital gyrus & 18 & -39 & -70 & -14 & 149 & 2.47 \\
\hline
\end{tabular}

Note: Minus sign represents a decrease ( $p<0.05$, AlphaSim corrected)

Abbreviation: MNI, Montreal Neurological Institute. 
Table 3 Regions Showing ALFF Differences Between ESRD and Control Groups

\begin{tabular}{|c|c|c|c|c|c|c|}
\hline \multirow[t]{2}{*}{ Brain Regions } & \multirow[t]{2}{*}{ Brodman Area } & \multicolumn{3}{|c|}{ MNI Coordinates (mm) } & \multirow[t]{2}{*}{ Volume $\left(\mathrm{mm}^{3}\right)$} & \multirow[t]{2}{*}{ Peak $t$ Value } \\
\hline & & $\mathbf{x}$ & $y$ & $\mathbf{z}$ & & \\
\hline Left parietal lobule & 41 & -45 & -25 & 8 & 41 & 3.29 \\
\hline Left Inferior parietal lobule & 40 & -42 & -24 & 9 & 37 & 3.29 \\
\hline Left superior temporal gyrus & 13 & -49 & -23 & 8 & 37 & 3.29 \\
\hline Left supramarginal Lobule & 42 & -42 & -18 & 8 & 31 & 3.29 \\
\hline Medial frontal gyrus & 6 & 3 & -3 & 54 & 52 & 4.74 \\
\hline Parahippocampal gyrus & 19 & 21 & -51 & -3 & 87 & 3.35 \\
\hline Left superior frontal gyrus & 9 & -18 & 36 & 39 & 74 & 2.56 \\
\hline Right superior frontal gyrus & 6 & 15 & 15 & 57 & 81 & 4.61 \\
\hline Right medial frontal gyrus & 9 & 9 & 48 & 24 & 41 & 3.10 \\
\hline
\end{tabular}

Note: Minus sign represents a decrease ( $p<0.05$, AlphaSim corrected).

Abbreviation: $\mathrm{MNI}$, Montreal Neurological Institute.

Firstly, patients with ESRD have significantly lower FA values than healthy control subjects in the medial frontal gyrus, parietal lobe, supramarginal gyrus, inferior parietal gyrus, and middle temporal gyrus.

Secondly, ALFF values in patients with ESRD were significantly decreased in the medial frontal gyrus, parietal lobe, inferior parietal gyrus, and parahippocampal gyrus, but increased in the bilateral superior frontal gyrus. These changes in nerve fibers and spontaneous neuron activity suggested an abnormality in the microstructure and restingstate brain function of patients with ESRD. As this study also showed, ESRD patients had lower hemoglobin levels, possibly resulting in anemia, which can induce brain perfusion elevation via cerebral vessel dilation to compensate for anemia-associated reduced rate of oxygen delivery to brain tissue in order to maintain necessary brain metabolism and function. However, the circulatory stress of routine hemodialysis can also cause cerebral vasculature damage, especially in older ESRD patients. ${ }^{35}$ The end results may be worsening endothelial dysfunction, negatively affecting cerebral vessel dilation as a compensation mechanism. A positron emission tomography (PET) study of $\mathrm{CO}_{2}$ response with small cohorts

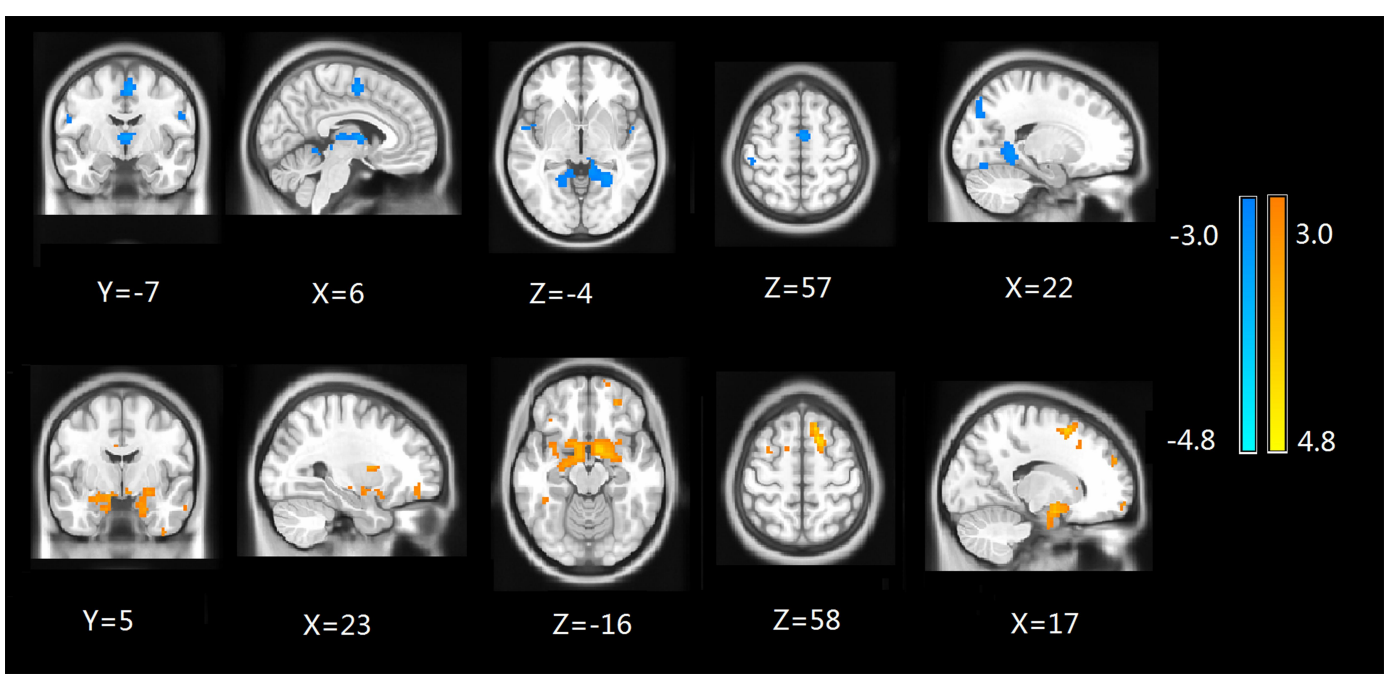

Figure 2 ALFF maps show differences between ESRD and control groups ( $p<0.05$, AlphaSim corrected). The patients with ESRD showed a significant ALFF decrease in the medial frontal gyrus, parietal lobe, inferior parietal gyrus, supramarginal gyrus, right lingual gyrus and parahippocampal gyrus (cold colors), but an increase in the bilateral superior frontal gyrus (warm colors). 

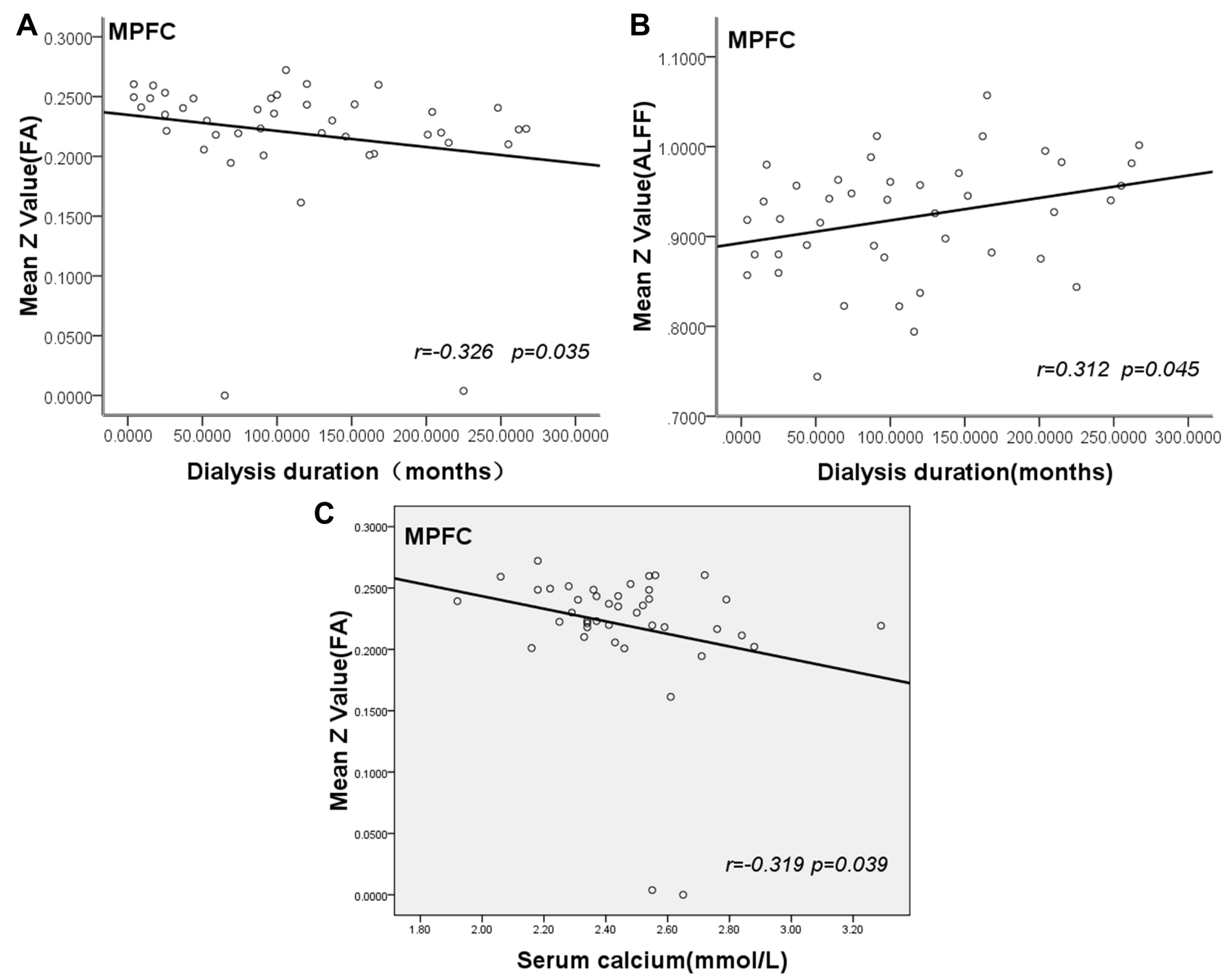

Figure 3 Scatter plot show correlation of dialysis duration with mean value of FA and ALFF in MPFC. The dialysis duration of ESRD patients negatively correlates with mean FA value in MPFC ( $r=-0.326, p=0.035)(\mathbf{A})$. Dialysis duration show positive correlation with mean ALFF value in MPFC ( $r=0.3$ I2, $p=0.045)(\mathbf{B})$. Serum calcium of ESRD patients negatively correlated with mean FA value in MPFC $(r=-0.319, p=0.039)(\mathbf{C})$.

Abbreviation: MPFC, medial prefrontal cortex.

of pre-hemodialysis and hemodialysis patients ${ }^{36}$ suggested reduced compensatory cerebral vessel dilation in hemodialysis patients. The reduced ability in compensatory cerebral vessel dilation can cause perfusion decline and inadequate oxygenation. This may explain the observed significant decline of ALFF. Thirdly, compared with the HCs, the ESRD patients had significantly lower MoCA and AVLTdelay recall scores, which indicated a decline in global cognitive function and memory. Interestingly, all of the brain areas with significant FA and ALFF changes were located in the frontal, temporal and parietal lobes. Frontal lobe dysfunction is characterized clinically by decreased abstraction, spontaneity, initiative, judgment, insight, perseverance, and response inhibition. ${ }^{37}$ Parietal cortex lesions are considered to be engaged in distinct attention functions such as visuospatial attention, attention shift, working memory, and supramodal control. ${ }^{38-40}$ The differences may contribute to a decline in cognitive function. Although no significant correlation was found between the neuropsychological tests and ALFF or FA values. The MoCA and AVLT neuropsychological tests used here only evaluated cognitive and memory function in general, and each specific cognitive ability was not assessed. So it cannot deny that the differences in ALFF or FA are associated with cognitive impairment. Some previous studies found that spontaneous neural activity in the special brain regions of DMN was correlated with number connection test-A (NCT-A) and digital symbol test (DST), which were associated with the domains of psychomotor speed, attention, and visual memory. So, more specific cognitive scales need to be added in the future. ${ }^{41}$ These regions 
are considered to be important components of human default-mode networks. ${ }^{42}$ The inferior parietal lobe is a major posterior component of the DMN. ${ }^{38,39}$ This is consistent with previous rs-fMRI studies based on functional connectivity $^{43}$ and independent component analysis methods ${ }^{14}$ in ESRD. Many previous studies ${ }^{18,44,45}$ have demonstrated abnormalities in brain structure and biochemistry of multiple DMN regions in ESRD patients.

Functionally, the DMN is engaged in a broad array of cognitive processing related to episodic memory, selfawareness, and interactive modulation between the internal brain activities and external tasks. ${ }^{42,46}$ Patients with ESRD often develop various cognitive deficits involving attention, executive function, and memory ${ }^{47,48}$ before any overt neurological manifestation. Thus, we speculated that the changed ALFF and FA values within-DMN might in part underlie these cognitive disturbances in ESRD. Our findings are similar to previous brain imaging study. ${ }^{12}$ Xue Liang et al investigated the spontaneous neural activity in ESRD patients by using rs-fMRI with regional homogeneity analysis (ReHo) algorithm. They found that ESRD patients show decreased ReHo in multiple areas of bilateral frontal, parietal, and temporal lobes. In addition, they found neuropsychological tests including NCT-A and DST, which were associated with the domains of psychomotor speed and domains of psychomotor speed, attention, and visual memory, were correlated with ReHo values in the special brain regions of DMN.

Furthermore, we found that the duration of hemodialysis in the ESRD group positively correlated with ALFF values in the bilateral superior frontal gyrus and negatively correlated with FA values in the medial frontal gyrus, parietal lobe, supramarginal gyrus, inferior parietal gyrus, and middle temporal gyrus. These results are different from those by Luo et $\mathrm{al}^{11}$ in which rs-fMRI with ALFF algorithm was applied to analyze the spontaneous brain activity patterns in ESRD patients undergoing peritoneal dialysis. This may be because different types of dialysis have different effects on brain function. Moreover, they found that line tracing test scores negatively correlated with ALFF values in bilateral middle temporal gyri and cuneus, and ALFF values in the right superior parietal lobe positively correlated with DST scores of patients in the PD group. A negative correlation was observed between serial dotting test scores and ALFF in the bilateral precuneus. But, they did not assess the global cognitive function. All the scales they used were associated with the domains of executive function. Therefore, a full cognitive battery measuring domain-specific cognitive function would be more informative and needed. We also found serum calcium of ESRD patients negatively correlated with FA values in the frontal and parietal lobes. The possible explanation is that many ESRD patients undergo dialysis, which is still not able to remove all the toxins, middlelarge molecules in particular, ${ }^{49}$ and, for unknown reasons, these toxins have been shown to particularly affect frontal white matter. The serum calcium level may play an important role in the integrity of the fiber bundle.

As well known in the literature, the majority of ESRD patients, including those in hemodialysis, are hypertensive. However, the cohort of HD patients in this study is not significantly different from the healthy control group in terms of blood pressure. As the ESRD patients and controls were randomized to have hypertension. Hypertension is very common in this age group, it is difficult to exclude the effect of blood pressure on results if only patients who do not have hypertension are selected.

We acknowledge several limitations to our study. First, the patients recruited in this study had heterogeneous etiologies, which may affect the statistical analysis and comprehensive interpretation of the results. Further studies with large-cohort and homogeneous etiology are needed. Second, this was a cross-sectional, but not a longitudinal study. A longitudinal study in patients with ESRD before and after hemodialysis is necessary to further investigate the pathophysiology of cognitive impairments in HD patients. Third, the differences in ALFF, FA, and MD between ESRD and control group may be partly related to ESRD itself and others were secondary to dialysis. Therefore, dialysis is possibly a crucial factor that leads to structural and functional impairment. Further studies about whether dialysis is requested and the pattern of dialysis nephropathy should be considered. Fourth, the MoCA and AVLT scale measured only global cognitive function and memory. A full cognitive battery measuring domain-specific cognitive function would be more informative. $^{41}$

\section{Conclusion}

Our study revealed both brain structure and function were impaired in the ESRD patients with routine hemodialysis, which distributed mainly in the parietal, temporal, and frontal lobes. ESRD patients had cognitive impairment and a decline in memory ability. The associations between ALFF, FA values and dialysis duration suggested that Serum calcium and dialysis duration might be associated 
with impairment of brain structure and function in patients with ESRD.

\section{Ethics Statement}

This prospective study was performed according to the Helsinki Declaration of 1975 as revised in 2013 and was approved by the Medical Research Ethics Committee of the Second Affiliated Hospital of Nanjing Medical University (protocol number: 2017KY082). Written informed consent was obtained from all subjects before the study.

\section{Acknowledgments}

This work was supported by a grant from Jiangsu Science and Technology Department of China (BE2017762) to Dr Junwei Yang.

\section{Disclosure}

The authors declare no competing financial interests and no conflicts of interest in this work.

\section{References}

1. de Deyn PP, Saxena VK, Abts H, et al. Clinical and pathophysiological aspects of neurological complications in renal failure. Acta Neurol Belg. 1992;92(4):191-206.

2. Brouns R, de Deyn PP. Neurological complications in renal failure: a review. Clin Neurol Neurosurg. 2004;107(1):1-16. doi:10.1016/j. clineuro.2004.07.012

3. Murray AM, Tupper DE, Knopman DS, et al. Cognitive impairment in hemodialysis patients is common. Neurology. 2006;67(2):216-223. doi:10.1212/01.wnl.0000225182.15532.40

4. Kurella Tamura M, Yaffe K. Dementia and cognitive impairment in ESRD: diagnostic and therapeutic strategies. Kidney Int. 2011;79 (1):14-22. doi:10.1038/ki.2010.336

5. Sehgal AR, Grey SF, Deoreo PB, et al. Prevalence, recognition, and implications of mental impairment among hemodialysis patients. $\mathrm{Am}$ J Kidney Dis. 1997;30(1):41-49. doi:10.1016/S0272-6386(97)90563-1

6. Bremer BA, Wert KM, Durica AL, et al. Neuropsychological, physi$\mathrm{cal}$, and psychosocial functioning of individuals with end-stage renal disease. Ann Behav Med. 1997;19(4):348-352. doi:10.1007/ BF02895153

7. Vogels SC, Emmelot-Vonk MH, Verhaar HJ, et al. The association of chronic kidney disease with brain lesions on MRI or CT: a systematic review. Maturitas. 2012;71(4):331-336. doi:10.1016/j.maturi tas.2012.01.008

8. Kuriyama N, Mizuno T, Ohshima Y, et al. Intracranial deep white matter lesions (DWLs) are associated with chronic kidney disease (CKD) and cognitive impairment: a 5-year follow-up magnetic resonance imaging (MRI) study. Arch Gerontol Geriatr. 2013;56 (1):55-60. doi:10.1016/j.archger.2011.11.009

9. Zhang LJ, Wen J, Ni L, et al. Predominant gray matter volume loss in patients with end-stage renal disease: a voxel-based morphometry study. Metab Brain Dis. 2013;28(4):647-654. doi:10.1007/s11011013-9438-7

10. Yu-Feng Z, Yong H, Zhu CZ, et al. Altered baseline brain activity in children with ADHD revealed by resting-state functional MRI. Brain Dev. 2007;29(2):83-91. doi:10.1016/j.braindev.2006.07.002
11. Luo S, Qi RF, Wen JQ, et al. Abnormal intrinsic brain activity patterns in patients with end-stage renal disease undergoing peritoneal dialysis: a resting-state functional MR imaging study. Radiology. 2016;278(1):181-189. doi:10.1148/radiol.2015141913

12. Liang X, Wen J, Ni L, et al. Altered pattern of spontaneous brain activity in the patients with end-stage renal disease: a resting-state functional MRI study with regional homogeneity analysis. PLoS One. 2013;8(8):e71507. doi:10.1371/journal.pone.0071507

13. Li C, Su HH, Qiu YW, et al. Regional homogeneity changes in hemodialysis patients with end stage renal disease: in vivo resting-state functional MRI study. PLoS One. 2014;9(2):e87114. doi:10.1371/journal.pone.0087114

14. Ni L, Wen J, Zhang LJ, et al. Aberrant default-mode functional connectivity in patients with end-stage renal disease: a resting-state functional MR imaging study. Radiology. 2014;271(2):543-552. doi:10.1148/radiol.13130816

15. Moodalbail DG, Reiser KA, Detre JA, et al. Systematic review of structural and functional neuroimaging findings in children and adults with CKD. Clin J Am Soc Nephrol. 2013;8(8):1429-1448. doi:10.2215/CJN.11601112

16. Chou MC, Hsieh TJ, Lin YL, et al. Widespread white matter alterations in patients with end-stage renal disease: a voxelwise diffusion tensor imaging study. AJNR Am $J$ Neuroradiol. 2013;34 (10):1945-1951. doi:10.3174/ajnr.A3511

17. Hsieh TJ, Chang JM, Chuang HY, et al. End-stage renal disease: in vivo diffusion-tensor imaging of silent white matter damage. Radiology. 2009;252(2):518-525. doi:10.1148/radiol.2523080484

18. Kim HS, Park JW, Bai DS, et al. Diffusion tensor imaging findings in neurologically asymptomatic patients with end stage renal disease. NeuroRehabilitation. 2011;29(1):111-116. doi:10.3233/NRE-20110684

19. Zhang LJ, Wen J, Liang X, et al. Brain default mode network changes after renal transplantation: a diffusion-tensor imaging and resting-state functional MR imaging study. Radiology. 2016;278 (2):485-495. doi:10.1148/radiol.2015150004

20. Nucifora PG, Verma R, Lee SK, et al. Diffusion-tensor MR imaging and tractography: exploring brain microstructure and connectivity. Radiology. 2007;245(2):367-384. doi:10.1148/radiol.2452060445

21. Alexander AL, Lee JE, Lazar M, et al. Diffusion tensor imaging of the brain. Neurotherapeutics. 2007;4(3):316-329. doi:10.1016/j. nurt.2007.05.011

22. Papanikolaou N, Karampekios S, Papadaki E, et al. Fractional anisotropy and mean diffusivity measurements on normal human brain: comparison between low-and high-resolution diffusion tensor imaging sequences. Eur Radiol. 2006;16(1):187-192. doi:10.1007/ s00330-005-2833-7

23. Ishida I, Hirakata H, Sugimori H, et al. Hemodialysis causes severe orthostatic reduction in cerebral blood flow velocity in diabetic patients. Am J Kidney Dis. 1999;34(6):1096-1104. doi:10.1016/ S0272-6386(99)70016-8

24. Gottlieb D, Mildworf B, Rubinger D, et al. The regional cerebral blood flow in patients under chronic hemodialytic treatment. $J$ Cereb Blood Flow Metab. 1987;7(5):659-661. doi:10.1038/jcbfm.1987.119

25. Hata R, Matsumoto M, Handa N, et al. Effects of hemodialysis on cerebral circulation evaluated by transcranial doppler ultrasonography. Stroke. 1994;25(2):408-412. doi:10.1161/01.STR.2 5.2 .408

26. Hoshino T, Ookawara S, Goto S, et al. Evaluation of cerebral oxygenation in patients undergoing long-term hemodialysis. Nephron Clin Pract. 2014;126(1):57-61. doi:10.1159/000358432

27. Postiglione A, Faccenda F, Gallotta G, et al. Changes in middle cerebral artery blood velocity in uremic patients after hemodialysis. Stroke. 1991;22(12):1508-1511. doi:10.1161/01.STR.22.12.1508

28. Cameron OG, Modell JG, Hariharan M. Caffeine and human cerebral blood flow: a positron emission tomography study. Life Sci. 1990;47 (13):1141-1146. doi:10.1016/0024-3205(90)90174-P 
29. Hachinski V, Iadecola C, Petersen RC, et al. National institute of neurological disorders and stroke-Canadian stroke network vascular cognitive impairment harmonization standards. Stroke. 2006;37 (9):2220-2241. doi:10.1161/01.STR.0000237236.88823.47

30. Yan $\mathrm{CG}$, Wang $\mathrm{XD}$, Zuo $\mathrm{XN}$, et al. DPABI: data processing \& analysis for (Resting-state) brain imaging. Neuroinformatics. 2016;14(3):339-351. doi:10.1007/s12021-016-9299-4

31. Song XW, Dong ZY, Long XY, et al. REST: a toolkit for resting-state functional magnetic resonance imaging data processing. PLoS One. 2011;6(9):e25031. doi:10.1371/journal.pone.0025031

32. Qi R, Zhang $\mathrm{L}, \mathrm{Wu} \mathrm{S}$, et al. Altered resting-state brain activity at functional MR imaging during the progression of hepatic encephalopathy. Radiology. 2012;264(1):187-195. doi:10.1148/ radiol.12111429

33. Power JD, Barnes KA, Snyder AZ, et al. Spurious but systematic correlations in functional connectivity MRI networks arise from subject motion. NeuroImage. 2012;59(3):2142-2154. doi:10.1016/j. neuroimage.2011.10.018

34. Power JD, Barnes KA, Snyder AZ, et al. Steps toward optimizing motion artifact removal in functional connectivity MRI; a reply to Carp. NeuroImage. 2013;76:439441.

35. Findlay MD, Dawson J, Dickie DA, et al. Investigating the relationship between cerebral blood flow and cognitive function in hemodialysis patients. J Am Soc Nephrol. 2019;30(1):147-158. doi:10.1681/ ASN.2018050462

36. Kuwabara Y, Sasaki M, Hirakata H, et al. Cerebral blood flow and vasodilatory capacity in anemia secondary to chronic renal failure. Kidney Int $\quad 2002 ; 61(2): 564-569$ doi:10.1046/j.15231755.2002.00142.x

37. Hirakata $\mathrm{H}$, Yao $\mathrm{H}$, Osato $\mathrm{S}$, et al. CBF and oxygen metabolism in hemodialysis patients: effects of anemia correction with recombinant human EPO. Am J Physiol. 1992;262(5 Pt 2):F737-43. doi:10.1152/ ajprenal.1992.262.5.F737

38. Lynch JC, Mountcastle VB, Talbot WH, et al. Parietal lobe mechanisms for directed visual attention. $J$ Neurophysiol. 1977;40 (2):362-389. doi:10.1152/jn.1977.40.2.362
39. Posner MI, Walker JA, Friedrich FJ, et al. Effects of parietal injury on covert orienting of attention. J Neurosci. 1984;4(7):1863-1874. doi:10.1523/JNEUROSCI.04-07-01863.1984

40. Mesulam MM. A cortical network for directed attention and unilateral neglect. Ann Neurol. 1981;10(4):309-325. doi:10.1002/ ana.410100402

41. Murray AM, Bell EJ, Tupper DE, et al. The brain in kidney disease (BRINK) cohort study: design and baseline cognitive function. Am J Kidney Dis. 2016;67(4):593-600. doi:10.1053/j.ajkd.2015.11.008

42. Buckner RL, Andrews-Hanna JR, Schacter DL. The brain's default network: anatomy, function, and relevance to disease. Ann N Y Acad Sci. 2008;1124:1-38.

43. Ma X, Tian J, Wu Z, et al. spatial disassociation of disrupted functional connectivity for the default mode network in patients with end-stage renal disease. PLoS One. 2016;11(8):e0161392. doi:10.1371/journal.pone.0161392

44. Tryc AB, Alwan G, Bokemeyer M, et al. Cerebral metabolic alterations and cognitive dysfunction in chronic kidney disease. Nephrol Dial Transplant. 2011;26(8):2635-2641. doi:10.1093/ndt/gfq729

45. Chiu ML, Li CW, Chang JM, et al. Cerebral metabolic changes in neurologically presymptomatic patients undergoing haemodialysis: in vivo proton MR spectroscopic findings. Eur Radiol. 2010;20 (6):1502-1507. doi:10.1007/s00330-009-1673-2

46. Fox MD, Snyder AZ, Vincent JL, et al. The human brain is intrinsically organized into dynamic, anticorrelated functional networks. Proc Natl Acad Sci U S A. 2005;102(27):9673-9678. doi:10.1073/ pnas.0504136102

47. Jassal SV, Roscoe J, Leblanc D, et al. Differential impairment of psychomotor efficiency and processing speed in patients with chronic kidney disease. Int Urol Nephrol. 2009;252(2):518-525. doi:10.1007/ s11255-008-9375-2

48. Williams MA, Sklar AH, Burright RG, et al. Temporal effects of dialysis on cognitive functioning in patients with ESRD. Am J Kidney Dis. 2007;245(2):367-384. doi:10.1053/j.ajkd.2003.12.031

49. Ronco C, La Greca G. The role of technology in hemodialysis. Contrib Nephrol. 2002;137:1-12.
The International Journal of Nephrology and Renovascular Disease is an international, peer-reviewed open-access journal focusing on the pathophysiology of the kidney and vascular supply. Epidemiology, screening, diagnosis, and treatment interventions are covered as well as basic science, biochemical and immunological studies. The manuscript management system is completely online and includes a very quick and fair peer-review system, which is all easy to use. Visit http://www.dovepress.com/testimonials.php to read real quotes from published authors. 